\title{
Student Attendance System based on the Face Recognition
}

\author{
Harshit Agarwal \\ $B C A$ \\ School of Computing Science and \\ Engineering (SCSE) \\ Jewar, India
}

\author{
Govinda Verma \\ $B C A$ \\ School of Computing Science and \\ Engineering (SCSE) \\ Agra, India
}

\author{
Lakshya Gupta \\ $B C A$ \\ School of Computing Science and \\ Engineering (SCSE) \\ Meerut, India
}

\begin{abstract}
Face Recognition Attendance system is important in schools and institutions. In school and institution is not easy to maintain the attendance records in paper and file. Therefore, an existing system that uses a face recognition attendance system increases accuracy and it takes less time as compared to another method. There are a lot of systems available such as face recognition using "IR motion" sensors, etc. With automatic face recognition attendance system, hardware devices(software), helpfull. But the challenge is to keep all the nerves properly without getting hurt. the face recognition attendance system we use javascripts,node.js and library are used tensorflow.js and face-API. It can take pictures from $50-70 \mathrm{~cm}$. We create a graphical interface that takes pictures, builds a database ,and trains the database with one click.
\end{abstract}

Before seeing student's face they store the data in an excel sheet with time and date and we can check the excel sheet it the and of the class and lecture for conformation.

\section{INTRODUCTION-}

As we've have seen in many schools and institutions, members of the institutions are faced with a problems of the presence of proxies, they keep all the handwritten notes to go to each classrooms each day. It's an awfully difficult task for them. To scale back their workload others strategies is emerging. Face recognition is the process of identifying something that has already been discovered as a known or unknown face. Often the problem of facial recognition is confusing and the problem of facial recognition Face recognition on the other hand determines whether a "face" is a well-known, or unknown, person who uses this purpose facial information to verify this facial expression.

\section{A. Literature Review-}

1) Attendance System based on face recognition-

The paper focus on improving school and college student attendance. Because there have been so many injustices in personal adoption as costs, illegal attendance and it can now no longer usually to be so. Biometric and floor strategies are consequently recognized. The traditional methods of seeing faces were not so bad. This paper represented the idea that saving time and improving the System. Face recognition attendance system procedures it's was easy. this sense the pictures was taken with a CCTV's camera at school / institutions with the intention of going there. He then first found the student features of the face and different facial expressions in the picture.

2) Smart Attendance Mgmt System Using Face Recognition

\section{Introduction-}

An primary idea of face popularity is photo process. There are kinds of photo process: Analogue Process: It's a method is help for difficult copy together with phot \&printing. computerized Performance: Controls virtual picture graph content material through PC. Basically attendance is of types: Student Arrival Program Automatic Visiting Program. There are many difficulties in manual attendance such as handling all the papers and noticing the attendance / absence from the pen every day, so it's required more struggle and longer. But the dis-advantages was cleared by the face recognition attendance method. A important purpose of the system is to get images of the student features(face), their location and appearance. There are more methods for attendance but they need some restrictions.

Biometric-based methods: It scans a specific part of the body like fingerprints $\&$ indicates an appearance. even this can take time. Hands free method: This system is not flexible and required up to 8 connections at once.

\section{3) Attendance Management with Facial Recognition}

Digital Scan: The student's fingerprint is scanned and keep in the data-collection. for register presence, students must apply a digital method after each lecture completed.

Keep card entry methods: The chip is placed on the student ID card, which is scanned by the system and reads the chip. That card will need to be scanned in each classroom to record attendance. A list of current and absent students is displayed to the faculty.

\section{4) Student's Attendance Marking Using Face} Recognition in IOT-

An original plan beyond the answer is to improve a face recognition attendance system. They reduces fake presence of the students and increases correctness.

In this adviced methods, facial recall was done by usedetector and read. The whole idea in the methods is divided into several stages-

At the started stage the student fill an registering form with all the points which were placed in the insitution datacollection. The student's picture / image is also placed in the data-collection. The steps's is require only one time.

Then, the classroom entrance should have a camera with sensors and microcontrollers. Here the "passive infrared detector". is use to quota emission from the objects. It also detects the movement of things. 
The first "passive infrared detector" measures the emission of an item and moving as the student enters the classroom. While the radiation is human range, the camera activates and captures images.

After the student clicks on the images it compared that pictures to the data-collection. If the pictures matches, they will update the data-collection.

With the present. Otherwise determined now no longer withinside the database. Every replace withinside the database approximately a selected student despatched to their dad and mom through e-mail or text each days. The machine additionally labored for school in equal manner. For school there has been every other data-collection had create. With the help of the machine we may further keep school.

\section{WORKING SYSTEM'S-}

Facial recognition Attendance System is an advanced application biometric software that uses an in-depth study methods to compared a live captured or image to a store person face print to confirm personal name. However, inintensity gaining knowledge of is a category of gadget gaining knowledge of algorithms that use more than one layers to steadily extract high-stage functions for an uncooked input For example, in image processing, the last line can detect the edges, while the upper line can detect human idea such as numbers or face.

Face recognition is the mehods of identify a person face in a scan picture to determine whether an object is human. We can use two applications -

1 - Node.js.

2- Node.js Express.

And in this system we can use also library for run a program. Library used are face-api.js ,Tensorflow.js and face landmark recognition web Api is used for feteching facial detection.

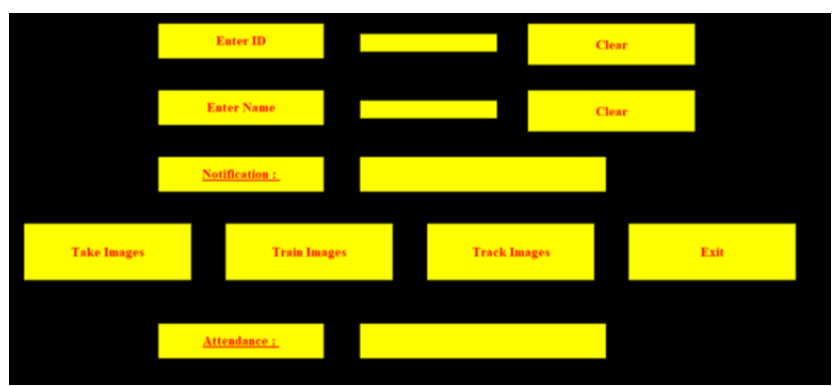

Fig. 1. Front page-

This was an gadget appearance show in, Fig1 indicates an gadget front page where in we's need to input roll no and title of pupil. After coming into the roll no and title of pupil we need to click on button named as Take's pictures. Then it'll take photograph a pupil .

\section{Facial Recognition App using TensorFlow.js}

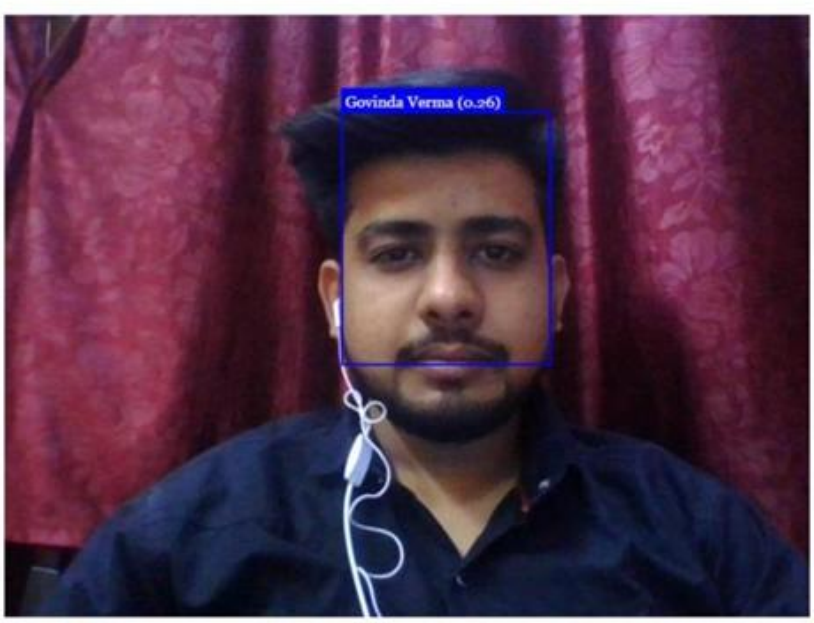

Fig. 2 .

Farther, we need a song picture. I need to click on on button names a Track pictures. Before monitoring unique picture display the call and roll no of pupil on his/her detected face as proven in upper picture. the statistics will saved in data-collection. After finishing all methods. At this stage we need to click on the Quit icon so that the student information is stored in the attendance as shown and it produces an excel file which is stored with the information of the students in the class. How the system works.

Let's understand the system by considering various scenarios to simplify it: class:

1. How the system works if only one student enters the

If a pupil enters the study room on time then the photographs of that unique pupil can be captured with the aid of using the digital digicam after which that picture can be educated to understand the students' faces primarily based totally at the photographs saved withinside the dataset.

Based on the facial recognition of the students as shown in the information related to that particular image, an Excel file will be generated and will store the students name, roll number, date and time. The system will then mark the students as current.

A records is saved withinside the device, the device is supposed to offer attendance to cutting-edge college students. 2. Consider the second one situation wherein many college students input the school room simultaneously. It works efficaciously even in that case. 


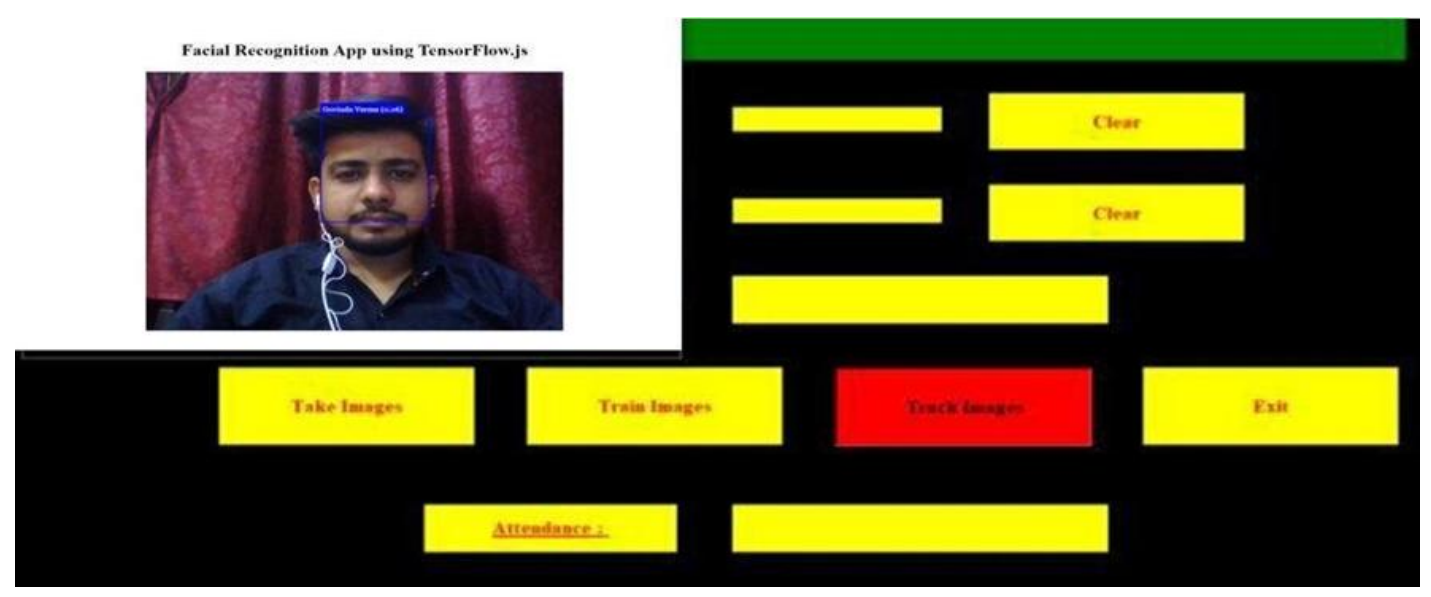

Fig. 3.

It can understand or understand a couple of faces whilst getting into the orbit, as proven withinside the photograph above. After detection, whilst we click on at the go out button it'll display the presence notification withinside the presence box. That presence is robotically saved withinside the attendance sheet with ID, name, date and time.

\section{RESULT-}

An records of pupil found in photograph saved in records. the gadget grip a couple of faces at in stage right. records was replace a proven a photograph.

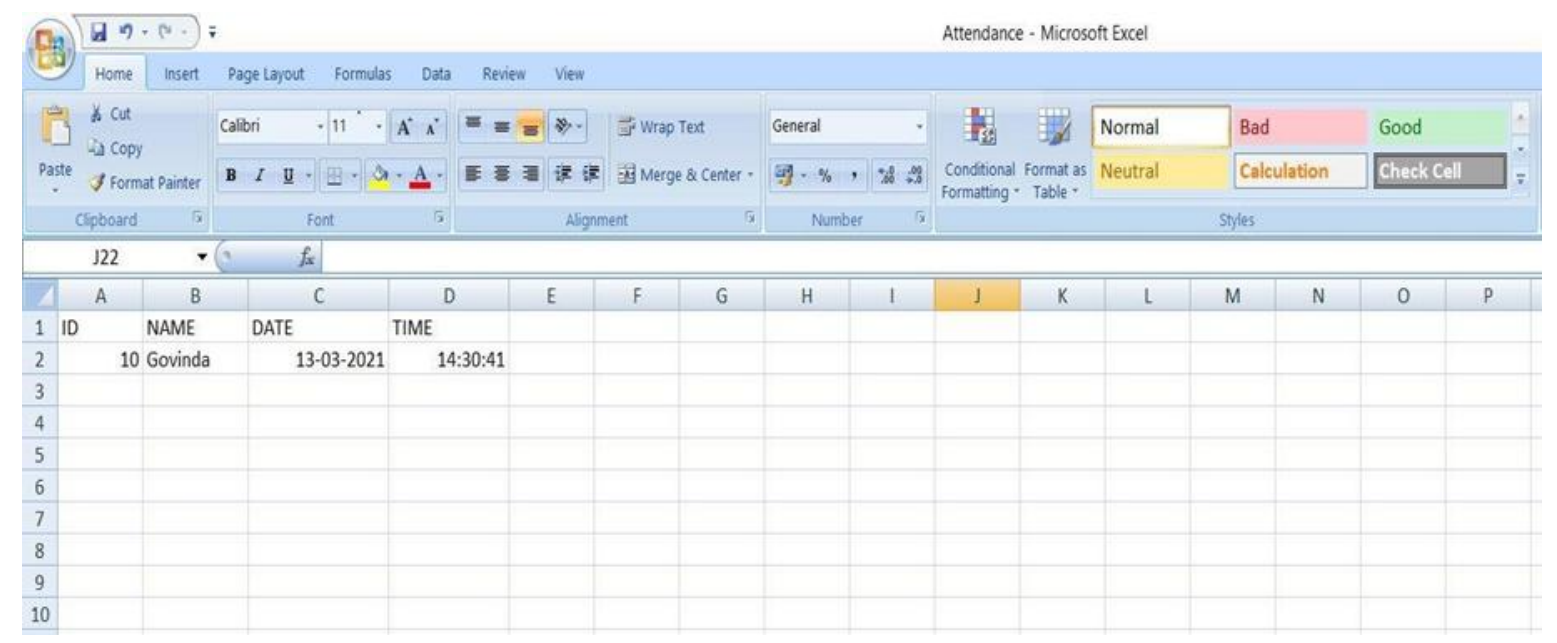

Fig. 4. Multiple students attendances has marked

Above Fig. indicates Information of pupil that have been saved into excel document after spotting their faces. It shops all statistics of pupil both it's miles unmarried face or more than one faces. System works nicely in all conditions. When pupil input into class (unmarried/more than one) in the event that they understand their identityentification and call saved in attendance sheet together with modern date and time.

\section{ADVANTAGES-}

1. It can take care of big databases and keep a big variety of pics for training.

2. The accuracy of this set of rules used is better than different algorithms.

3. It is able to shooting pics very correctly from a distance of 60-eighty $\mathrm{cm}$.

4. Suppose if a scholar by accident enters a couple of roll numbers on the time of face popularity then it suggests all of the roll numbers withinside the output.
5. Network connectivity isn't always required so there might be no community associated problems.

6. The time it takes to create a dataset and educate an picture could be very short.

7. Human and system direct interface is much less and which reduces many mistakes and will increase the accuracy to a top notch extent.

8. The gadget is straightforward to function for the duration of scholar attendance and is likewise smooth to function with precise accuracy.

9. The gadget works well for lots faces and look updates successfully.

\section{FUTURE SCOPE-}

Therefore it will be very helpful to maintain the appearance automatically with the help of facial recognition and the chances of errors will be less as compared to manual process. This will also reduce the rigging of attendance records of students and will also save time. 
The system can be configured to detect fraud and be used in ATM machines. In addition, the system can be used at the time of useelections where the voter can be identified by recognizing the face.

\section{CONCLUSION-}

In this paper, we tend to studied the use of deep neural networks on face detection and recognition. We tend to analyzed some of the different neural networks including convolutional Neural networks, depthwise separable convolutions, and densely linked convolutional networks. We enforced an easy implemented a simple face detection and recognition system using Node.js and TensorFlow.js core API making use of existing API like the face-api.js package located

https://github.com/justadudewhohacks/face-api.js/. This library is optimized to be used in the browser. Generating the data is out of the scope of this research, but there are publicly available datasets that can be used to load in the app for doing face recognition. The API allowed us to run different experiments where users can detect the emotion of a person, the gender, and the age of a person. In the last years, facial recognition has been well developed that now we can build apps that can recognize faces with great accuracy. The fascinating half concering this experiment is to seek out additional helpful application for this technology .

\section{REFFERENCES-}

[1] S. Min, B. Lee, and S. Yoon, "Deep learning in bioinformatics," Briefings in Bioinformatics, 2016.

[2] G. Dahl, N. Jaitly, "Multi-task Neural Networks for QSAR Predictions," University of Toronto, 2014.

[3] K. Zhang, Z. Zhang, Z. Li and Y. Qiao, "Joint Face Detection and Alignment Using Multitask Cascaded Convolutional Networks," in IEEE Signal Processing Letters, vol. 23, no. 10, pp. 1499- 1503, Oct. 2016. doi: 10.1109/LSP.2016.2603342.

[4] S. I innainmaa "The renresentation of The cumulative rounding blunders of an set of rules as a Taylor growth of the local rounding errors," Master's thesis, Univ. Helsinki, 1970.

[5] P. J. Werbos, "Beyond Regression: New Tools for Prediction and Analysis in the Behavioral Sciences," PhD thesis, Harvard University, 1974.

[6] J. Yu and C. Li, "Face Recognition Based on Euclidean Distance and Texture Features," 2013 International Conference on Computational and Information Sciences, Shiyang, 2013, pp. 211- 213. doi: 10.1109/ICCIS.2013.63.

[7] T. Guo, J. Dong, H. Li and Y. Gao, "Simple convolutional neural network on image classification," 2017 IEEE 2nd International Conference on Big 